\title{
5 A psicoeducação e a sobrecarga dos cuidadores informais do idoso com demência: análise baseada na evidência
}

Francisco Miguel Correia Sampaio

\section{RESUMO}

Ademênciaéumapatologianeurodegenerativaemininterrupto crescimento na população portuguesa, como resultado da diminuição da natalidade e aumento da esperança média de vida, sendo indiscutíveis as suas repercussões, quer para o sujeito, quer para os seus cuidadores. A psicoeducação é uma intervenção de Enfermagem que compreende intervenções sistémicas, didácticas e psicoterapêuticas, adequadas para informar o doente e os seus familiares acerca da doença e do seu tratamento, facilitando a compreensão e a gestão responsável da doença, e dando suporte a todos aqueles que se vêm obrigados a lidar com a doença.

Sabendo que a síndrome demencial afecta gravemente, de forma indirecta, os cuidadores informais, este artigo visa perceber se a psicoeducação é uma intervenção de Enfermagem efectiva para reduzir a sobrecarga dos mesmos. Para o efeito, recorreu-se à revisão bibliográfica de três revisões sistemáticas, num modelo assente nos pressupostos da Enfermagem Baseada na Evidência.

Da análise realizada, verificou-se que apenas uma das revisões sistemáticas apresentava dois estudos que sugeriam que a psicoeducação poderia estar na origem de uma ligeira redução da sobrecarga dos cuidadores informais, sendo que as outras duas revisões sistemáticas alvo de análise foram unânimes na conclusão da ineficácia da psicoeducação, com uma ausência na redução da sobrecarga.

Assim, a psicoeducação parece não ser efectiva, mas verificase uma ausência de consenso acerca da intervenção, sendo necessário realizar mais investigação na área, sobretudo em contexto nacional, onde existem ainda poucos estudos efectuados, tendo sido apenas encontrado um trabalho de Mestrado, cujas conclusões eram até opostas àquelas que foram encontradas nas revisões sistemáticas internacionais analisadas.

PALAVRAS-CHAVE: Sobrecarga; Psicoeducação; Prestador de Cuidados; Demência.

\section{ABSTRACT}

Dementia is a neurodegenerative disease that is continuously increasing in Portuguese population as a result of birth rate diminution and increasing average life expectancy, being incontestable its repercussions to the patient and to his/ her caregivers. Psychoeducation is a Nursing intervention that comprises systemic, didactic-psychotherapeutic interventions, which are adequate for informing patients and theirs relatives about the illness and its treatment, facilitating both and understanding and personally responsible handling of the illness and supporting those afflicted in coping with the disorder.

Knowing that dementia syndrome affects, indirectly, the informal caregivers, this article aims to understand if psychoeducation is an effective Nursing intervention to reduce caregivers burden. To do so, and using bibliographic review, there were reviewed three systematic reviews through an Evidence-Based Nursing model.

The analysis showed that only one of the systematic reviews presented two studies that suggested psychoeducation could help to slightly reduce caregivers burden. However, the other analysed systematic reviews suggested that psychoeducation presents no efficacy, as caregivers' burden did not decrease as a result of psychoeducation programmes.

So, the intervention seems not to be effective, but there is an absence of consensus about that, being necessary to carry out more investigation in this area, mainly in national context, as there was found only one Master's Dissertation whose conclusions were totally opposite to the conclusions found in the analysed international systematic reviews.

\section{KEYWORDS: Burden; Psychoeducation; Caregiver; Dementia.}

\section{INTRODUÇÃO}

Ao longo dos tempos, e com a crescente afirmação da Enfermagem enquanto disciplina científica, vai sendo crescente a necessidade de uma prestação de cuidados suportada pela evidência científica. Este caminho torna

\footnotetext{
${ }^{1}$ Enfermeiro, Domus Care Porto / Mestrando em Enfermagem de Saúde Mental e Psiquiatria, fmcsampaio@gmail.com Submetido em 26-03-2011. Aceite em 31-05-2011.

Citação: Sampaio, F. M. C. (2011) A psicoeducação e a sobrecarga dos cuidadores informais do idoso com demência: análise baseada na evidência. Revista Portuguesa de Enfermagem de Saúde Mental, 5, 30-36.
} 
possível a argumentação acerca das práticas clínicas com base em estudos que demonstram a utilidade, eficácia e eficiência de uma dada intervenção (possivelmente em detrimento de outra).

No contexto específico da Saúde Mental e Psiquiatria, existem ainda diversas intervenções e, sobretudo, modalidades de intervenção psicoterapêutica cuja aplicação é considerada, inclusivamente por alguns profissionais de saúde, como sendo secundária. Nesse sentido, o presente artigo visa analisar a pertinência da psicoeducação dos cuidadores informais do idoso com demência, como forma de perceber se esta intervenção pode ser útil para a redução da sobrecarga dos mesmos.

A escolha da demência, e em particular dos prestadores de cuidados, deve-se ao facto de esta ser uma patologia que tem vindo a afectar cada vez mais idosos, estimando-se que em Portugal, no ano de 2010, existam cerca de 70 mil doentes com Doença de Alzheimer (Leuschner, 2005, Cit. por Sequeira, 2007). A intervenção nos prestadores de cuidados visa a prevenção da transição saúde/doença dos mesmos, na medida em que estes, pelas dificuldades associadas à prestação de cuidados, acabam por sofrer, frequentemente, de patologias como a depressão e a ansiedade (Garrido e Almeida, 1999, Garrett, 2005, Cit. por Sequeira, 2007).

Ao nível metodológico, serão analisadas três revisões sistemáticas da literatura com o objectivo de verificar a utilidade da psicoeducação dos prestadores de cuidados da pessoa com demência na diminuição da sobrecarga dos mesmos sendo, a posteriori, e pelo confronto com a prática clínica, analisada a possibilidade da implementação da intervenção aos nível da prestação de cuidados domiciliários.

Numa fase inicial do presente trabalho, e visto que este irá versar sobre a psicoeducação, torna-se imperioso, antes de mais, definir claramente este conceito. Assim, o termo psicoeducação começou por ser utilizado por Anderson et al. (1980, Cit. por Baüml, Froböse, Kraemer, Rentrop \& Pitschel-Walz, 2006), de forma a descrever um conceito terapêutico comportamental consistindo em quatro elementos: informar o doente relativamente à sua doença; treino de solução de problemas; treino de comunicação; treino de auto-assertividade. Apesar de a psicoeducação ser dirigida, primariamente, para o doente, os familiares também poderiam ser incluídos.

A partir da metade da década de 1980 um entendimento independente da psicoeducação começou a surgir nos países de língua alemã (Baüml et al., 2006). Assim, o grupo de trabalho "Psychoeducation of Patients with Schizophrenia" (Baüml \& Pitschel-Walz, 2003, Cit. por Baüml et al., 2006) formulou uma definição em que a psicoeducação era entendida como compreendendo intervenções sistémicas, didácticas e psicoterapêuticas, adequadas para informar o doente e os seus familiares acerca da doença e do seu tratamento, facilitando a compreensão e a gestão responsável da doença, e dando suporte a todos aqueles que se vêm obrigados a lidar com a doença. Não tendo a estrutura da psicoterapia, a psicoeducação refere-se aos componentes do tratamento em que a comunicação activa de informação, a partilha de informação com todos as pessoas implicadas no processo, e o tratamento de aspectos gerais da doença são proeminentes.

Asindicaçõespara a participação num grupopsicoeducacional são bastante alargadas. Por outro lado, existem apenas escassas contraindicações, incluindo distúrbios massivos do pensamento formal, humor eufórico (fase maníaca), alucinações auditivas de comando, e ideação suicida aguda, geralmente com uma resiliência ao stress reduzida. Os doentes podem ser integrados no tratamento desde que tenham a capacidade de tomar parte num grupo durante um período de cerca de 60 minutos (no caso de a psicoeducação ser realizada em grupo) (Baüml et al., 2006)

\section{DEFINIÇÃO DA PERGUNTA DE PARTIDA (MODELO PICO)}

A prática baseada na evidência implica o seguimento de cinco passos (etapas) que têm início na formulação da questão de partida, e que culminam na avaliação do desfecho (avaliação da aplicabilidade prática da evidência identificada) (Gray, 2004).

Segundo o mesmo autor (Ibidem), todos os encontros clínicos geram questões; no entanto, nem todas elas podem encontrar resposta no cliente ou numa fonte colateral do mesmo, na medida em que se relacionam com o processo de cuidar da pessoa e, portanto, apenas podem ser respondidas na literatura adequada. Este tipo de questão é mais bem estruturado, sendo o modelo de quatro partes (PICO) um dos mais utilizados para a formulação da mesma. Assim, a questão deve incluir o cliente ou a pessoa visada; a intervenção visada, incluindo qualquer grupo de comparação; o resultado de interesse (Badenoch \& Heneghan, 2002, Dawes, 1999, Geddes, 1999, McKibbon et al., 2002, Sackett et al., 2000, Cit. por Gray, 2004). As questões PICO são assim denominadas porque incluem uma ajuda mnemónica das palavras: população, intervenção, comparação, e resultados (Gray, 2002, Cit. por Gray, 2004).

O presente trabalho visa perceber os efeitos que a psicoeducação tem ao nível da sobrecarga dos cuidadores informais do idoso com demência. Neste sentido, e seguindo o modelo PICO, a população serão os cuidadores informais (do idoso com demência), a intervenção será a psicoeducação, não existe qualquer tipo de comparação, já que se pretende avaliar os efeitos da psicoeducação per si, e não por comparação com qualquer outro tipo de intervenção de Enfermagem, e o resultado a verificar será a diminuição da sobrecarga (dos cuidadores informais do 
idoso com demência). Assim, e seguindo o modelo PICO, a questão de partida para o presente trabalho é: será que a psicoeducação permite a diminuição da sobrecarga dos cuidadores informais do idoso com demência?

Após a formulação de uma questão PICO, a etapa seguinte do processo da prática baseada na evidência é a busca pelas principais evidências que permitam dar resposta a essa questão (Gray, 2004), sendo o capítulo seguinte dedicado a essa mesma etapa.

\section{REVISÃO DA LITERATURA}

Numa fase inicial da etapa de busca por respostas, é importante que a revisão da literatura, antes de realizada, seja devidamente planeada, pelo que é fundamental criar uma lista de palavras a pesquisar (Beaven, in Craig \& Smyth, 2004). Assim, e seguindo os itens do modelo PICO, as palavras pesquisadas foram: caregivers e dementia (população); psychoeducation (intervenção); burden (resultados). A opção pela pesquisa na língua inglesa (apesar de também ter sido realizada em português, ainda que com resultados que foram rejeitados dada a sua relativa irrelevância) deve-se ao facto de existir um maior número de resultados disponíveis em inglês, permitindo ainda o acesso a trabalhos mais relevantes (por exemplo, guidelines e revisões sistemáticas da literatura).

Após estar perfeitamente delineado o planeamento da revisão da literatura, deve iniciar-se a execução da mesma. Para tal, uma abordagem bastante eficiente é a estratégia hierárquica dos " 5 passos" de Haynes, que envolve sistemas, sumários, sinopses, sínteses e estudos (Haynes, 2006).

O ponto de partida para a busca deve ser o que Haynes denominou por sistema (Haynes, 2001a, 2001b, Cit. por Gray, 2004), sendo que é nos sistemas que podem ser encontradas as guidelines (directrizes práticas clínicas) (Gray, 2004). No entanto, e apesar de terem sido encontradas algumas guidelines ligadas à demência, e à prestação de cuidados ao idoso com demência, nenhuma delas fazia referência clara aos efeitos directos da psicoeducação na redução da sobrecarga do cuidador informal do idoso com demência. $\mathrm{Na}$ pesquisa efectuada, foram apenas encontradas duas guidelines que referiam a utilidade da psicoeducação no cuidador informal da pessoa com demência (American Academy of Neurology, 2001), e na redução da depressão do cuidador informal e existência de efeitos moderados da intervenção múltipla (na qual se incluía a psicoeducação) na redução da sobrecarga e melhoria do bem-estar do cuidador informal (National Collaborating Centre for Mental Health, 2007). No entanto, não seria possível perceber se esses efeitos estariam associados apenas à psicoeducação, ou ao somatório das diversas intervenções realizadas concomitantemente.
Considerando que não foi possível o acesso a qualquer resumo ou sinopse, passou-se de imediato para o nível seguinte de evidência: as sínteses (Gray, 2004). A este nível foram encontradas duas revisões sistemáticas e uma meta-análise que abordavam, todas elas, os resultados decorrentes de diversas intervenções junto dos cuidadores informais do idoso com demência, tendo sido verificados resultados que apresentavam a relação existente entre a psicoeducação e a sobrecarga do cuidador informal (Acton \& Kang, 2001; Schulz, O’Brien, Czaja, Ory, Norris, Martire, et al., 2002; Thompson, Spilsbury, Hall, Birks, Barnes \& Adamson, 2007).

\section{AVALIAÇÃO CRÍTICA DA LITERATURA}

Numa fase inicial da avaliação crítica da literatura encontrada (neste caso apenas revisões sistemáticas), importa perceber a qualidade da mesma, na medida em que até as revisões sistemáticas de fontes respeitadas podem apresentam problema metodológicos (Hopayian, 2001, Olsen et al., 2001, Cit. por Gray, 2004), pelo que é importante que essas revisões passem pela avaliação do leitor.

Assim, a primeira questão que deve ser colocada é: a revisão trata de uma questão claramente definida? (Gray, 2004). No caso das revisões sistemáticas em análise, verificase que o trabalho "Interventions to Reduce the Burden of Caregiving for an Adult With Dementia: A Meta-Analysis" (Acton \& Kang, 2001), não apresenta uma questão PICO explícita, podendo assumir-se que esta seja: qual o efeito das estratégias de intervenção (grupo de suporte, educação, psicoeducação, aconselhamento, descanso do cuidador e intervenções múltiplas) na redução da sobrecarga dos cuidadores informais do membro da família com demência. Pode afirmar-se que o tema, ainda que seja amplo (dada a multiplicidade de intervenções analisadas), permite uma revisão bem sustentada.

Ainda analisando o mesmo trabalho, verifica-se que os critérios de inclusão estão perfeitamente especificados, sendo que os autores (Acton \& Kang, 2001) referem, com base na literatura, que as meta-análises devem ser inclusivas, pelo que decidiram analisar todos os dados disponíveis, sem excluir qualquer tipo de estudo (excepto aqueles que não incluíam a sobrecarga enquanto resultado). Os estudos em análise parecem ser apropriados para dar resposta à questão identificada pelos autores. A qualidade dos estudos incluídos foi apreciada, tendo sido seguidos os critérios de qualidade desenvolvidos por Verhagen et al. (1998) e Brown (1991) - escala entre 0 e 21 . Os artigos analisados apresentavam uma variação na escala entre 7 e 20 , sendo contudo notória uma predominância de artigos com uma avaliação mínima de 14/15. Ainda no mesmo trabalho, os resultados dos diferentes estudos foram combinados e apresentados sob a forma de tabela, o que permitiu verificar a existência de uma relativa homogeneidade entre os artigos, com a devida 
explicação relativamente às diferenças nos resultados (por exemplo, existência de diferentes escalas de avaliação da sobrecarga, e de uma sobrecarga objectiva e subjectiva). Os efeitos de cada tipo de intervenção foram apresentados com os respectivos intervalos de confiança. As conclusões do trabalho foram sustentadas pelos dados do mesmo, tendo sido realizada uma explanação exaustiva acerca das diferenças encontradas e da validade da sobrecarga do cuidador informal como indicador de resultado.

Relativamente ao trabalho "Dementia Caregiver Intervention Research: In Search of Clinical Significance" (Schulz et al., 2002), a finalidade da revisão foi claramente formulada, ainda que não sob a forma de questão PICO. Assim, esta revisão sistemática pretendia reunir artigos em que eram abordados diversos indicadores de resultado relativos ao prestador de cuidados da pessoa com demência, tentando perceber quais as intervenções que estavam por detrás, e qual a eficácia e relevância das mesmas. Pode afirmar-se que se trata de um trabalho bastante amplo, ou até mesmo demasiado amplo, já que pretende analisar não só as intervenções psicossociais, mas também as intervenções ambientais, comportamentais, e até mesmo farmacológicos (neste caso, apenas para o receptor dos cuidados). Os critérios de inclusão e de exclusão (estudos de caso e relatos qualitativos, ou dados descritivos) estão expressos no trabalho, crendo-se igualmente que estes foram apropriados, dado o rigor apresentado para a selecção dos mesmos. Contrariamente ao trabalho referido anteriormente, este não apresenta uma apreciação da qualidade dos estudos incluídos, apesar de terem sido usados critérios explícitos na selecção dos mesmos. Os resultados dos estudos foram apresentados na forma de tabela mas, dada a natureza do estudo, em que era avaliada a variação dos indicadores de resultado, mais do que os efeitos de cada intervenção específica, não foi possível apresentar uma combinação dos resultados dos estudos, nem fazer uma análise profunda da heterogeneidade dos mesmos, ainda que tenham sido apresentadas algumas razões para justificar as diferenças encontradas nos resultados do estudo. As conclusões do trabalho foram fundamentadas pelos dados, tendo sido ainda realizada uma comparação entre os resultados obtidos e as informações que haviam sido sugeridas pela literatura mais recente.

Finalmente, o trabalho "Systematic review of information and support interventions for caregivers of people with dementia" (Thompson et al., 2007) apresenta a finalidade da revisão claramente formulada, ainda que não esteja presente sob a forma de questão PICO. No entanto, a questão subjacente ao trabalho está relacionada com os efeitos das intervenções relacionadas com o suporte e a educação nos prestadores de cuidados da pessoa com demência (abarcando uma diversidade de indicadores de resultados, ligados ao cuidador, ao doente, à utilização de serviços de saúde e resultados ao nível económico). Pode assim afirmar-se que se trata de um trabalho amplo, mas não em demasia dado que apenas se avaliam os efeitos de dois tipos de intervenção. Os critérios de inclusão encontram-se claramente especificados, sendo que os autores referem mesmo o tipo de estudo apropriado para responder à questão: ensaio clínico aleatório controlado. Para além disso, é exposta uma estratégia para identificar estudos relevantes, sendo referidas as bases de dados pesquisadas, bem como as palavras-chave utilizadas. Foi ainda realizada uma apreciação detalhada da qualidade dos estudos incluídos, sendo feita referência a critérios explícitos (critérios de inclusão e adequação do processo de aleatoriedade). Porém, os autores referem que, a nível geral, os estudos incluídos apresentam uma qualidade pobre, na medida em que as estratégias de aleatoriedade eram pouco claras e ocultação da distribuição era rara. Os resultados dos estudos foram apresentados sob a forma de tabela e de gráfico, havendo evidência de alguma heterogeneidade, mas não ao nível de todos os tipos de intervenção. Os resultados dos estudos incluídos foram combinados, mas apenas foram discutidas superficialmente as razões das diferenças nos resultados, dado que os autores consideram que a evidência produzida não apresenta uma qualidade indiscutível. As conclusões apresentadas pelos autores foram fundamentados pelos dados obtidos pela análise dos estudos, tendo ainda sido realizada uma análise da aplicabilidade da evidência identificada.

A meta-análise realizada por Acton e Kang (2001) incluía, dentro da categoria da psicoeducação, as intervenções educacionais e de suporte, pelo que os participantes que pertenciam aos grupos de tratamento recebiam estes dois tipos de intervenção. Assim, a psicoeducação integrava, por exemplo, o fornecimento de informação estandardizada acerca do processo de doença, dos comportamentos disruptivos e dos skills para a prestação de cuidados, bem como actividades de suporte desenhadas para fazer uso do diálogo em grupos de ajuda mútua para partilhar experiências, e aumentar as ligações com os membros do grupo. De acordo com esta meta-análise, a intervenção múltipla (que excluía a psicoeducação) apresentou um ligeiro efeito positivo na sobrecarga do cuidador informal. Contudo, a psicoeducação não teve efeitos positivos significativos ao nível da sobrecarga. Assim, este trabalho sugere que as intervenções usadas não tiveram qualquer efeito na sobrecarga, sendo que algumas delas tiveram mesmo um efeito negativo, ou os scores de sobrecarga do grupo de controlo apresentaram melhorias superiores aos do grupo de tratamento.

A revisão sistemática apresentada por Schulz et al. (2002) faz referência a um estudo realizado em 1999 por Belmin et al. que sugere uma diminuição do risco de sobrecarga (de moderado para baixo) em prestadores de cuidados submetidos a um programa educacional de 3 semanas em pequenos grupos. É ainda feita referência a um estudo realizado em 2000 por Gallagher-Thompson et al. que sugere que alguns prestadores de cuidados, a realizar uma intervenção psicoeducacional de satisfação com a vida, referiram uma diminuição significativa da sobrecarga. Segundo Schulz et al. (2002), existem algumas intervenções 
que podem ser efectivas para a redução da sobrecarga, pelo menos em alguns prestadores de cuidados. No entanto, o significado prático destes resultados é discutível.

Finalmente, a revisão sistemática realizada por Thompson et al. (2007) refere que três estudos de intervenção psicoeducacional (231 participantes), que incluía o fornecimento de informação e de suporte, avaliaram a sobrecarga e que não foram encontrada diferenças significativas entre os grupos (de controlo e de tratamento). Assim, os mesmos autores (Ibidem) referem que a revisão realizada sugere a inexistência de evidência quanto à efectividade das intervenções informativas e de suporte para os cuidadores das pessoas com demência.

De acordo com Soares (2005), os níveis de evidência podem ser divididos usando uma numeração de I a VII (I - revisão sistemática; II - ensaio clínico randomizado; III - Coorte; IV - caso-controle; V - séries de casos; VI opinião de especialistas; VII - estudos pré-clínicos). Assim, pode verificar-se que o material encontrado para servir de base à análise se classifica como apresentando um nível de evidência de grau I. Newman \& Roberts (in Craig \& Smyth, 2004) apresentam uma hierarquia de evidência extremamente semelhante relativamente a questões sobre a eficácia de uma dada intervenção ou terapia, pelo que se pode afirmar que a evidência encontrada (em revisões sistemáticas, com ou sem meta-análise) é a melhor para dar resposta à questão inicialmente formulada.

\section{INTEGRAÇÃO DA EVIDÊNCIA NA PRESTAÇÃO DE CUIDADOS}

A prestação de cuidados ao nível domiciliário, através de uma empresa privada, é um meio privilegiado para integrar qualquer tipo de intervenção baseada na educação, na medida em que não se verificam questões ligadas à falta de tempo nem de apoio institucional. Porém, podem existir alguns défices ao nível dos recursos (já que as empresas visam a obtenção de lucros máximos em função de gastos mínimos), bem como de autoridade para efectuar mudanças já que, ao nível dos cuidados de saúde de cariz privado, a designação "cliente" ganha uma lógica muito superior à de "utente/doente", sendo o objectivo fundamental a não perda do cliente, que poderá ocorrer se este se manifestar descontente com o tipo de intervenção implementado.

Fazendo uma análise das revisões sistemáticas incluídas neste trabalho, verifica-se que todas elas são bastante inclusivas e não exclusivas, característica extremamente importante para a aplicabilidade prática da evidência, na medida em que os doentes e os locais de um estudo nunca são idênticos àqueles em que se trabalha (Newman \& Roberts, in Craig \& Smyth, 2004). Nas revisões sistemáticas analisadas, não foi possível perceber alguns factores chave para a possível afectação dos resultados, como a idade dos participantes, género, etc. Contudo, e dada a grande inclusividade dos estudos, é crível que as intervenções tenham sido implementadas em prestadores de cuidados de pessoas com demência de diversos tipos e com diversas características particulares.

Relativamente à introdução da intervenção (psicoeducação) no meio de trabalho (prestação de cuidados domiciliários através de empresa privada), esta é perfeitamente possível, já que a empresa dispõe de recursos humanos suficientes para uma implementação óptima da intervenção, considerando ainda que esta não teria custos significativos. Porém, e apesar da aparente ausência de custos, coloca-se uma questão bastante pertinente quando se pensa nos benefícios: será que a implementação da psicoeducação terá efeitos positivos na diminuição da sobrecarga do cuidador informal do idoso com demência? A verdade é que as revisões sistemáticas analisadas foram praticamente unânimes na verificação de uma não efectividade da intervenção, pelo que esta poderia ser rejeitada pela ausência de evidência científica (dentro da pesquisa realizada) que comprove a eficácia da mesma. No entanto, é importante perceber que os estudos incluídos nas revisões sistemáticas não foram realizadas em Portugal, pelo que pode existir um viés decorrente da realização dos estudos em realidades diferentes (ainda que não existam significativas variações socioculturais, já que os países de onde provieram as amostras eram todos eles ocidentais e desenvolvidos).

Para a aplicação da intervenção (psicoeducação), importa ainda perceber a vontade do cliente (Newman \& Roberts, in Craig \& Smyth, 2004), já que a evidência não é o único factor que informa a tomada de decisão clínica. Assim, é importante perceber o que significam os resultados destes estudos para o contexto de trabalho e para os clientes com os quais se desenvolve o processo terapêutico.

Finalmente, é extremamente importante perceber se a qualidade dos estudos (neste caso, das revisões sistemáticas) é suficientemente boa para utilizar os resultados (Newman \& Roberts, in Craig \& Smyth, 2004). Neste caso particular, e após já ter realizado uma avaliação prévia da qualidade das revisões sistemáticas para análise, pode afirmar-se que, ainda que estas apresentem algumas insuficiências, referidas pelos próprios autores, a sua aplicabilidade prática não pode ser questionada devido à sua qualidade já que, dentro das condicionantes existentes, a evidência produzida é de boa qualidade.

Em jeito de conclusão, pode afirmar-se que uma empresa privada de prestação de cuidados domiciliários apresenta boas condições para a implementação de uma intervenção ligada à psicoeducação dos prestadores de cuidados informais dos idosos com demência. Para além disso, a boa qualidade das revisões sistemáticas analisadas (apesar de estarem longe da excelência por apresentarem algumas limitações, sobretudo ao nível metodológico) e a abertura de uma boa parte dos clientes a este tipo de intervenções 
fariam com que estivessem reunidas condições óptimas para a implementação da intervenção. No entanto, a evidência encontrada contraria a utilidade da psicoeducação na redução da sobrecarga dos cuidadores informais dos idosos com demência, o que tornaria a integração da intervenção na prestação de cuidados bastante complexa, tanto para a empresa (que em termos de custo-benefício não vislumbra vantagens significativas) como para os profissionais de Enfermagem (que não possuem evidência que lhes faça parecer útil a implementação da psicoeducação nos cuidadores informais de idosos com demência). Apesar de tudo, deve considerar-se a não especificação das características biopsicossociais dos cuidadores informais estudados, bem como a realização dos estudos em contextos socioculturais algo diferentes da realidade portuguesa.

\section{CONCLUSÃO}

A análise baseada na evidência realizada teve como principal objectivo a validação da importância da psicoeducação enquanto intervenção de Enfermagem que permitisse a diminuição da sobrecarga dos cuidadores informais do idoso com demência. Para tal, procedeu-se à análise de três revisões sistemáticas (com ou sem meta-análise) seleccionadas em função da sua qualidade e adequação à temática em estudo.

Os resultados obtidos, apesar de não poderem ser excessivamente generalizados, considerando as limitações dos estudos analisados bem como os diferentes contextos socioculturais, apontam para a não efectividade da psicoeducação na diminuição da sobrecarga do cuidador informal, na medida em que apenas uma das revisões sistemáticas analisadas (com apenas dois estudos relacionados com a intervenção) apresenta alguma melhoria por parte dos cuidadores informais, sendo que as restantes duas sugerem que não existem quaisquer benefícios advindos da implementação da intervenção, chegando mesmo a verificar-se, em alguns estudos, um ligeiro aumento da sobrecarga do prestador de cuidados. Porém, no caso de haver necessidade da realização da intervenção (psicoeducação) no contexto das empresas privadas de cuidados domiciliários, parecem estar reunidas as condições para tal, excepto se for verificada escassez de tempo para a realização das visitas domiciliárias.

Fazendo uma comparação entre os resultados obtidos e os objectivos inicialmente propostos pode afirmar-se a eficácia do presente trabalho, ainda que com as devidas limitações, dado que as revisões sistemáticas analisadas apresentam algumas limitações, sobretudo pela grande inclusividade dos estudos analisados. Pode ainda afirmar-se a eficiência deste trabalho, considerando a escassez de tempo (que impediu a realização da última etapa da Prática Baseada na Evidência - avaliação da implementação da intervenção) e até mesmo de recursos para pesquisa, na base da realização do mesmo.
Concluindo, e por análise dos resultados obtidos, a psicoeducação parece não ser uma intervenção efectiva para a redução da sobrecarga dos cuidadores informais do idosos com demência. Porém, sugere-se a realização de estudos mais específicos, preferencialmente no contexto de Portugal, onde apenas foi encontrado um estudo realizado por Silva (2009), com resultados bastante distintos dos obtidos nas revisões sistemáticas internacionais analisadas, de modo a verificar se, efectivamente, as particularidades do contexto sociocultural influenciam os resultados obtidos.

\section{REFERÊNCIAS BIBLIOGRÁFICAS}

Acton G. J., \& Kang J. (2001). "Interventions to reduce the burden of caregiving for an adult with dementia: a metaanalysis". Research in Nursing \& Health, 24 (5), pp. 349-360. Acedido em 12 de Dezembro de 2010. Disponível em: http:// web.ebscohost.com/ehost/detail?hid $=10 \&$ sid $=5 \mathrm{bff} 46 \mathrm{eb}$ 3302-4253-8d06-1624a561f755\%40sessionmgr111\&vid=7.

American Academy of Neurology (2001). "Practice parameter: Management of Dementia (An Evidence-Based Review)". Neurology, 56, pp. 1154-1166. Acedido em 9 de Dezembro de 2010. Disponível em: http://faculty.ksu.edu.sa/daif/ Neurology\%20Fellowship/demntia\%20for\%20fellow.pdf.

Baüml, J., Froböse, T., Kraemer, S., Rentrop, M., \& PitschelWalz, G. (2006). "Psychoeducation:ABasicPsychotherapeutic Intervention for Patients with Schizophrenia and Their Families". Schizophrenia Bulletin, 32 (S1), pp. S1 - S9. Acedido em 17 de Março de 2011. Disponível em: http:// schizophreniabulletin.oxfordjournals.org/content/32/suppl_ 1/S1.full.pdf+html.

Beaven, O. (2004). Pesquisa de Literatura. In Craig, J. V., \& Smyth, R. L. - Prática Baseada na Evidência: Manual para Enfermeiros. Loures: Lusociência, pp. 47-85.

Gray, G. E. (2004). Psiquiatria Baseada em Evidências. Porto Alegre: Artmed.

Haynes, R. B. (2006). "Of studies, syntheses, synopses, summaries, and systems: the " $5 \mathrm{~S}$ " evolution of information services for evidence-based health care decisions". ACP Journal Club, 145 (3), pp. A8-A9. Acedido em 10 de Janeiro de 2011. Disponível em: http://web.ebscohost.com/ehost/det ail hid $=18 \&$ sid $=5$ bff 46 eb $-3302-4253-8 d 06-1624 a 561 f 755 \%$ 40 sessionmgr $111 \&$ vid $=5 \&$ bdata $=$ JnNpdGU9ZWhvc3QtbGl2 $Z Q \% 3 d \% 3 d \# d b=a 9 h \& A N=23245673$ National Collaborating Centre for Mental Health (2007). Dementia: A NICE-SCIE Guideline on supporting people with dementia and their carers in health and social care. Leicester/London: The British Psychological Society/The Royal College of Psychiatrists. Acedido em 15 de Dezembro de 2010. Disponível em: URL: http://www.nice.org.uk/nicemedia/pdf/CG42Dementiafinal. pdf. 
Newman, M., \& Roberts, T. (2004) - Apreciação Crítica 1: É a qualidade do estudo suficientemente boa para utilizar os resultados? / Apreciação Crítica 2: Pode a evidência ser aplicada no seu contexto?. In Craig, J. V., \& Smyth, R. L. - Prática Baseada na Evidência: Manual para Enfermeiros. Loures: Lusociência, pp. 86-135.

Schulz, R., O’Brien, A., Czaja, S., Ory, M., Norris, R., Martire, L. M. et al. (2002). "Dementia Caregiver Intervention Research: In Search of Clinical Significance". The Gerontologist, 42 (5), pp. 589-602. Acedido em 5 de Dezembro de 2010]. Disponível em:http://www.rosalynncarter.org/UserFiles/In\% 20 Search\%20of\%20Clinical\%20Significance.pdf.

Sequeira, C. (2007). Cuidar de Idosos Dependentes: Diagnósticos e Intervenções ( ${ }^{a}$ Ed.). Coimbra: Quarteto.
Silva, A. M. (2009). Efectividade de um programa de intervenção na percepção de sobrecarga dos familiares cuidadores de pessoas idosas com demência. Dissertação de Mestrado em Cuidados Paliativos. Lisboa: Universidade de Lisboa, Faculdade de Medicina de Lisboa.

Thompson, C.A., Spilsbury, K., Hall, J., Birks, Y., Barnes, C., \& Adamson, J. (2007). "Systematic review of information and support interventions for caregivers of people with dementia". BMC Geriatrics, 7, pp. 18. Acedido em 20 de Dezembro de 2010. Disponível em: http://web.ebscohost.com/ehost/ pdfviewer/pdfviewer?hid=12\&sid=5bff46eb-3302-42538d06-1624a561f755\%40sessionmgr111\&vid=15. 